\title{
Evaluation of a servo settling algorithm
}

\author{
Brian A. Bucci ${ }^{\mathrm{a}}$, Jeffrey S. Vipperman ${ }^{\mathrm{a}, *}$, Daniel G. Cole ${ }^{\mathrm{a}}$, Stephen J. Ludwick ${ }^{\mathrm{b}}$ \\ ${ }^{a}$ University of Pittsburgh, 636 Benedum Hall, 3700 O'Hara St. Pittsburgh, PA 15261 \\ ${ }^{b}$ Aerotech Inc., 101 Zeta Drive, Pittsburgh, PA 15238
}

\begin{abstract}
The aim of this work is to discuss methods of friction identification and provide experimental evaluation of a novel control algorithm that enhances settling after point-to-point motion. This algorithm is called the Nonlinear Integral Action Settling Algorithm or NIASA. As the name suggests, the integral gain is nonlinear, and is based upon a Dahl friction model. The settling resulting from PID+NIASA control is nearly exponential, and governed by a time constant that is specified in the control design. As the NIASA algorithm requires, friction parameters must be identified for the servo under test. Two methods of friction identification (Step Tests and Identification Profile) are contrasted and found to provide comparable results, although the latter can provide advantages. The identified friction parameters are in turn used to perform four sets of control experiments; Two PID controllers (standard factory tuning and high performance PID with acceleration feedforward) are tested both with and without NIASA compensation. In the case study with a factory tuned PID controller, servo settling times to within \pm 3 to $\pm 100 \mathrm{~nm}$, are reduced by between $80.5 \%$ and $87.4 \%$ when NIASA compensation is added. When the NIASA compensator is added to the high performance PID controller, servo settling time is still reduced by between $50.5 \%$ and $73.0 \%$. Although the NIASA compensator was designed to increase settling performance for relatively large point-to-point motions, similar positive results are achieved when the method is applied to smaller step motions that do not leave the pre-rolling friction regime. Frequency domain analyses demonstrated the nonlinear loop-gain of the plant, with a clear distinction between the rolling and pre-rolling friction cases. As expected, the nonlinear loop gain was found to lower the bandwidth for smaller motions. Adding NIASA control was observed to increase the bandwidth for small motions by a factor of 3-6, while having little effect on for large motions.
\end{abstract}

Keywords: friction, friction compensation, point-to-point motion, nonlinear control

\section{Running Headline: Servo settling algorithm}

Key words: friction, friction compensation, point-to-point motion, nonlinear control

\section{Introduction}

The behavior of the force of friction has long been known to cause problems in precision motion applications. In efforts to describe the issues caused by friction, advanced friction models, such as the Dahl model [1], LuGre model [2], and generalized Maxwell-slip model [3], have been developed. These advanced friction models are capable of reproducing the hysteretic behavior observed in real friction data [4].

This work aims to address the problem of reducing the time required to settle to nanometer level accuracy with a precision servo mechanism. While this work uses advanced hysteretic friction models, its direction is somewhat different from more widely accepted methods of friction compensation. Brief mention of these accepted methods of friction compensation will be made. However, the focus of this discussion is to state how such

\footnotetext{
${ }^{*}$ Corresponding author Tel.: 1412624 1643; Fax: 412-624-4846

Email address: jsv@pitt.edu (Jeffrey S. Vipperman)
}

methods are appropriate for other motion control problems, but they are not appropriate for this particular problem.

One widely accepted method of friction compensation is friction model feedforward. With a known trajectory and model of the friction process, friction can be predicted and partially compensated by feedforward control [5]. In profile tracking applications, tracking performance can often be significantly improved $[3,5,6,7,8,9,10,11,12]$. In tracking applications, the position reference has dynamics which are passed through the friction model in attempt to cancel the force of friction. However, during settling, the servo's position reference is usually frozen to a static value. Therefore, feedforward friction compensation will have little to no effect on servo settling [5].

Another accepted method of friction compensation is the friction observer. Friction observers have been successfully implemented with most significant advanced friction models $[2,5,11,13,14,15,16,17,18]$. Several adaptive extensions have also been investigated [19, 20, 21, 22, 23]. In many previous efforts, friction observers are shown to improve servo tracking performance $[2,5,14,15]$. However, experimental results from some of these same authors suggest that friction observers may not improve servo point-to-point performance but rather, they may actually degrade it $[11,13]$. In their paper on an adaptive friction observer, Canudas de Wit and Lischinsky [13] go on to state that regular integral action is probably more useful 
for point-to-point motion than is the friction observer.

The most simple and practical way to achieve steady state error specifications is to include some controller integral action. The Yosida Nano-Mechanism Project presents the first case of a direct drive linear stage which is able to achieve nanometer precision with a single actuator [24, 25]. This project represents a very similar situation that is being studied in the current effort. The key result from Futami et al. is that integral control was a major factor in achieving nanometer level positioning precision.

In previous work by the authors, an algorithm for settling servo mechanisms to nanometer level tolerances is proposed [26, 27, 28]. This method is called the Nonlinear Integral Action Settling Algorithm (NIASA). Some strengths of this method are its robustness to modeling errors in the friction process and how closely the proposed method relates to conventional PID tuning. In this work, the NIASA compensator will be briefly described. This will be followed by a discussion of how friction affects point-to-point motion. Next, parameterization of the NIASA compensator and friction model identification with be discussed. Then, the results of an example identification study and an actual point-to-point motion study will be presented. Finally, familiar frequency domain analysis will be used to describe the nonlinear controlled and uncontrolled systems at specific points of operation.

\section{Control Algorithm}

This section is designed to briefly introduce the proposed NIASA control algorithm to the reader. For further description of the methodology used to design the NIASA algorithm the reader is referred to the references $[27,28]$. In a brief summary, the NIASA compensator is an extension of classical PID control, where the controller integral action is modified by a friction model. Integral action plays a key role in achieving nanometer level servo precision [24]. However, use of integral action can slow the response of the system and carries the risk of causing limit cycles [12, 29, 30]. Thus, this work aims to design the controller integral action to quickly achieve nanometer level precision, while avoiding undesirable characteristics.

The simplified equation of motion for a controlled servo, subject to friction, is

$$
m \ddot{x}+F_{r}=u(t),
$$

where $m$ is the mass or moment of inertial, $x$ is displacement, $F_{r}$ is the friction process, and $u(t)$ is the control signal. PID control can be expressed as

$$
u(t)=k_{P} e+k_{I} \int_{0}^{t} e(t) d t+k_{D} \dot{e}
$$

where $e$ is the position error and $k_{P}, k_{I}$, and $k_{D}$ are the respective PID gains. For the servo mechanisms studied in this work the Dahl model has proved sufficient to describe the friction process. The Dahl model, in differential form, can be stated as

$$
\frac{d F_{r}}{d x}=\sigma\left(1-\frac{F_{r}}{F_{C}} \operatorname{sgn} \dot{x}\right)^{i},
$$

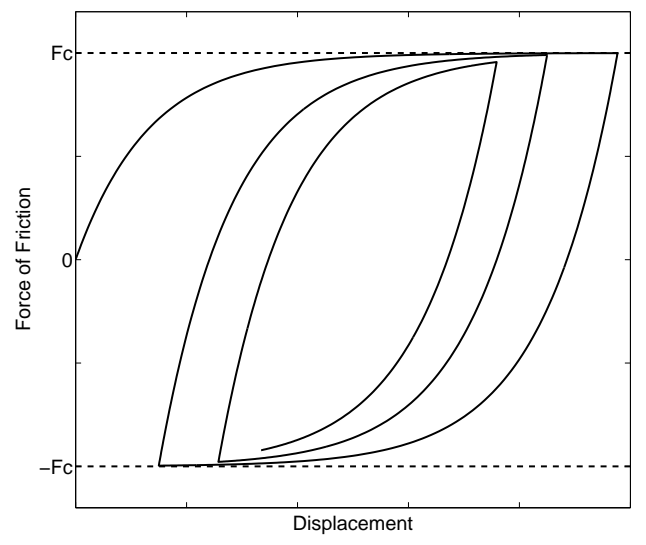

Figure 1: Example of the type of response seen from Dahl's friction model to a decaying sinusoidal motion.

where the differential $\frac{d F_{r}}{d x}$ is the change in the value of the force of friction versus change in displacement, $\sigma$ is the initial contact stiffness, $F_{C}$ is the level of Coulomb friction, and $i$ is a shape factor typically set to $i=1$. Figure 1 shows a graphical example of the behavior of the Dahl model. In figure 1 the simulated data starts at zero force on the left side of the plot. As the simulated system moves to the right, the force of friction increases and approaches the Coulomb level. When a velocity reversal occurs, at the upper right corner of the plot, a sharp transition in the force of friction is observed. As motion continues to the left, the force of friction approaches the opposing level of Coulomb friction. This pattern continues and the loops are traversed in the clockwise direction. The shape of each new transition curve that is initiated upon velocity reversal depends upon the friction state when the reversal occurs. Thus, the system has memory back to the last velocity reversal. Considering the behavior of the system described in figure 1, suppose the system starts at an arbitrary location with an arbitrary initial friction state. Suppose that it is desired to move the system to a second arbitrary location. The final friction state will depend on the initial friction state and the path to the desired location. Further, the final friction state could be a non-zero value. In this case, integral action is typically used to allow a servo under linear control to provide the necessary holding force to achieve zero steady state error.

In Bucci et. al [27, 28], the NIASA control law of

$$
u(t)=\int_{0}^{t}\left(\frac{1}{\tau_{d}} \frac{d \hat{F}_{r}}{d x}+k_{I}\right) e(t) d t+k_{P} e+k_{D} \dot{e}
$$

is proposed, where $\tau_{d}$ is a design time constant and $\frac{d \hat{F}_{r}}{d x}$ is the modeled differential of the friction process described by equation (3). The respective gains $k_{P}, k_{I}=k_{P} / \tau_{d}$, and $k_{D}$ are the gains produced from the PID tuning.

The NIASA control law is designed to have an ideal closed loop response, governed by the design time constant, $\tau_{d}$. Introducing the $\frac{d \hat{F}_{r}}{d x}$ term has the effect of linearizing the closed loop system. This additional nonlinear integral gain term will 
have a response similar to figure 1 after velocity reversal. Note that if we set $\frac{d \hat{F}_{r}}{d x}=0$, the system reverts back to standard PID tuning, which is referred to as the "base PID" controller. Both the integral gain and the friction differential term are scaled by the reciprocal of $\tau_{d}$.

Most simply described, this control law uses larger integral gains when very near to a velocity reversal, while traversing the steep part of the hysteresis curve. As the system moves further from velocity reversal the integral gain is reduced to maintain stability. The strength of this method is that it does not rely on an extremely accurate friction model or complex adaption. Conversely, a static parameterization, where parameters need only to be in the neighborhood of their true values, appears to offer significant improvement in point-to-point servo performance. The remaining sections of this document will focus on a brief discussion of how friction affects point-to-point servo motion, parameterizing the NIASA control law, friction identification, and studying the point-to-point performance of the proposed compensator.

\section{Friction and Point-to-Point Motion}

Linear systems theory and linear controls work very well to predict and achieve system performance, provided that the system under consideration is linear. In the case of a servo mechanism with rolling contacts, friction introduces a nonlinearity into the system. In this section, we will explain some of the artifacts that friction produces from the perspective of linear control design. Many control software packages use loop transmission plots and swept-sine analysis to identify the plant and aid in tuning. Generally, the actuator used in a high precision servo would be capable of providing forces far greater than any friction in the bearings; otherwise, the servo would not be able to move effectively. This often leads to control design being based mainly around controlling the inertia of the mechanism, a linear system.

Figure 2 shows an example of loop transmission plot from a precision servo where the amplitude of the identification signal is equal to $5 \%$ of the maximum output of the amplifier, which is 10 amps peak. Examining figure 2, this system could be estimated to have a frequency cross-over near $100 \mathrm{~Hz}$ with a phase margin of approximately 35 degrees. From this data, an approximate linear (free mass + PD control) closed loop system could be constructed. Figure 3 shows the response of this ideal system compared to an actual $100 \mu \mathrm{m}$ step response of a precision servo. An initial examination of figure 3 would indicate that the simple linear model appears to capture the behavior of the servo rather well. However, if figure 3 is re-scaled to focus on the final micrometer of servo settling, as seen in figure 4, it is apparent that the simple linear model does not offer a global description of the servo behavior.

The cause of this difference is pre-rolling friction. It is known from the previous discussion of the Dahl model that friction changes most dramatically very near velocity reversals. When the servo is identified using linear system identification methods, a sinusoid is input to the system and, ideally, the same
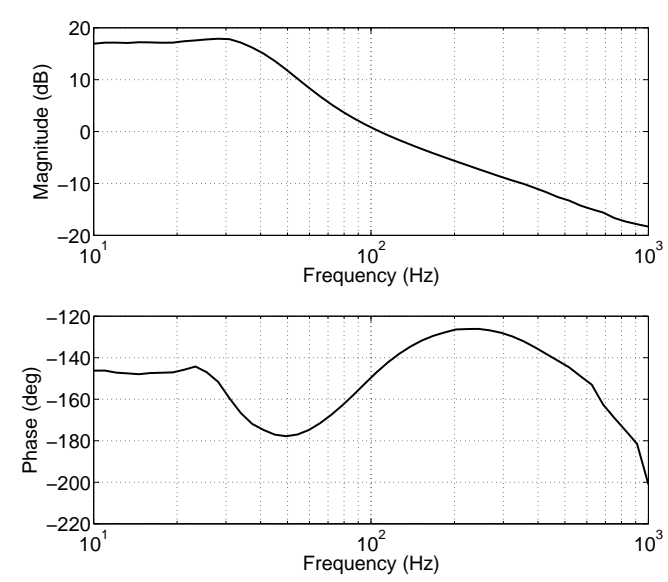

Figure 2: A loop transmission plot for the first PID controller shows the system to have a cross-over frequency near $100 \mathrm{~Hz}$ and a phase margin of about 35 degrees.

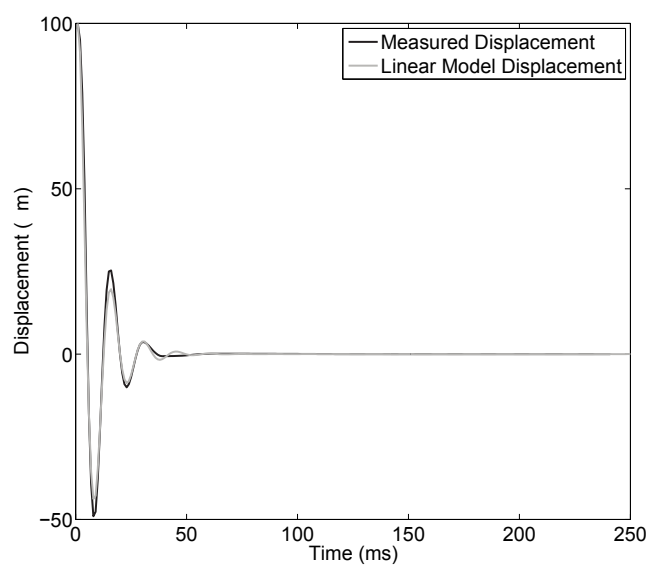

Figure 3: Comparison of actual $100 \mu \mathrm{m}$ step response to that predicted by a linear model.

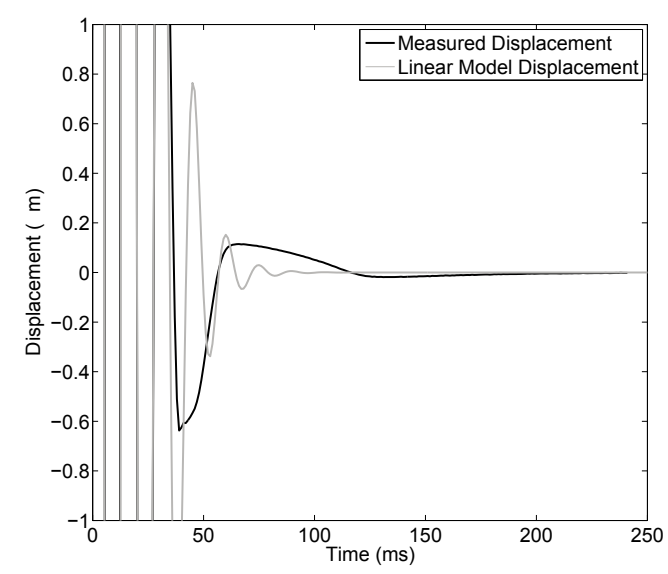

Figure 4: Final micrometer of settling demonstrating that the system response is dominated by the nonlinearity of pre-rolling friction. 

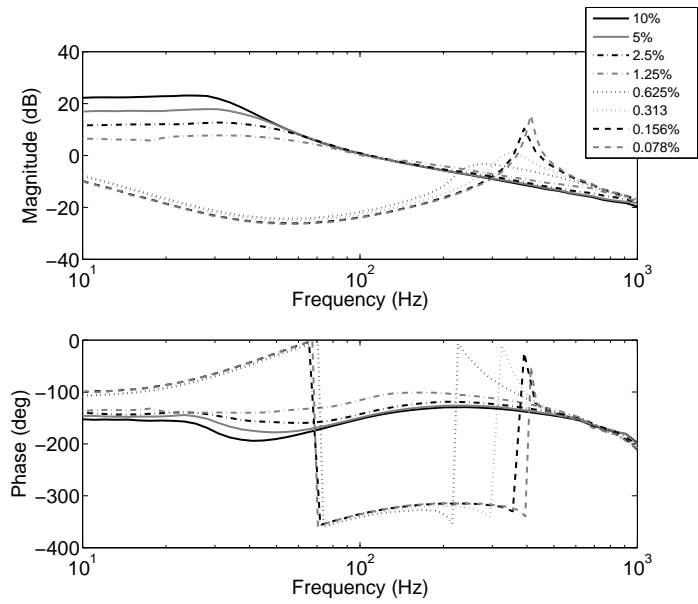

Figure 5: Loop transmission curves for various drive ratios of the plant.

frequency sinusoid is output from the system, with a change in magnitude and phase. For a linear system, the change in magnitude, at a given frequency, should be independent of the amplitude of the input signal. However, friction nonlinearity does create an amplitude dependence in the loop-gain. Thus, loop transmissions can appear very different depending upon how far the servo moves during identification. In figure 5, a family of loop transmission plots for $10 \%, 5 \%, 2.5 \%, 1.25 \%$, $0.625 \%, 0.313 \%, 0.156 \%$, and $0.078 \%$ of the maximum amplifier output, for a precision servo using the same PID controller, is presented.

In examining the data presented in figure 5 , it is seen that all of the series are different. These different data sets can be divided into two broad categories. For excitation amplitudes of $1.25 \%$ of maximum output and greater, the loop transmission is more represenative of what may be expected of a simple servo mechanism. Although there are differences in magnitude at the lower frequencies, the frequency domain behavior near frequency cross-over is similar and the cross-over frequency is also very similar, when comparing the data sets to each other. These data sets would suggest that the loop-gain has a crossover frequency of approximately $100 \mathrm{~Hz}$. For excitation amplitudes of $0.625 \%$ of maximum output and smaller, the apparent servo bandwidth has drastically decreased and a resonance has appeared just above $400 \mathrm{~Hz}$. The reason for these two distinct groups of data is friction.

In the case of the higher amplitude excitation data, the force input to the system exceeded the level of Coulomb friction. Thus, the servo traveled greater distances and more energy went into the inertial response of the system. For the lower amplitude excitation data, the input force was less than the level of Coulomb friction. In this case, the servo remains in the sticking condition (prerolling friction regime) and travels much less distance. Recalling the behavior of the Dahl model, for very small motions, friction can appear to act as a stiffness (with some damping). The scale of these small motions is typically less than the characteristic length of the friction-dominated regime in either direction, $x_{c}=F_{c} / \sigma$, or approximately $70 \mathrm{~nm}$ for the system used in this study. Since the servo has mass, for small amplitude excitations, the loop gain plots appear to have a resonance which is due to friction. These observations from linear systems identification methods agree remarkably well with theoretical describing function analysis performed by Helmick and Messner [31].

Use of linear methods in analyzing a nonlinear system can provide a means of understanding servo settling. Since the lower amplitude identification tests have velocity reversals very close to each other, this data could be thought of as describing the system when very close to a velocity reversal. Conversely, the higher amplitude identification tests have velocity reversals further from each other so, this data could be thought of as describing the system when it is further from velocity reversal. Thus, immediately after velocity reversal occurs, the servo loop could be approximated by the loop gain from the lowest drive ratio in figure 5. As motion proceeds in the same direction, the system transitions into the behavior seen in the higher driveratio loop transmissions. The degree of this transition is related to the distance from the velocity reversal. In the process of servo settling, velocity reversals occur at decreasing spatial intervals. Thus, the effective servo bandwidth tends to decrease as settling proceeds, and slowing of the settling process is observed.

\section{NIASA Control Law Parameterization}

The first step in implementing the NIASA compensator given by equation (4) is to construct a simple linear PID tuning for the servo mechanism. The base PID tuning should be able to complete the point-to-point motion operation within specifications such as in-position noise, however it may not be able to reach this condition within the specified time constraint. The NIASA compensator becomes most useful in time sensitive, ultra-precision applications. The next section will outline experimental identification of the friction term given in equation (3).

\section{Friction Identification}

With a base PID tuning, the next step in constructing the NIASA compensator is friction identification $\left(F_{C}\right.$ and $\sigma$ in equation (3)). One of the difficulties in this stage is determining how much of the frictional behavior needs to be modeled. Therefore, the length scale where pre-rolling effects significantly influence the system response must be determined. Two different methods of friction parameter identification will be presented next and contrasted.

\subsection{Step Response Test}

Recalling that pre-rolling friction has the greatest influence on system response near velocity reversals, this process aims to ascribe a value to the notion of "near a velocity reversal". This is done by injecting the same motion profile into the actual system and the linear representation and comparing their responses. A profiled step in reference position that produces a 


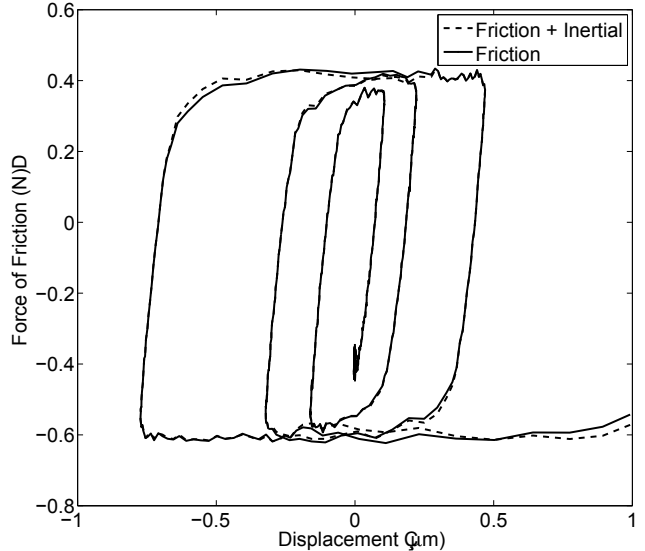

Figure 6: Force versus displacement curve generated by the identification profile.

number of velocity reversals while keeping within the amplifier limits is used for friction parameter identification.

As the oscillations from the step response decay, at some distance from the target location, the data from the real step response should significantly deviate from the ideal linear approximation. This is the regime where the servo response is dominated by pre-rolling friction. A key feature that can be used to differentiate two regimes is the time between the local minimums and maximums of the response. When the system is well approximated as a linear system the local minimums and maximums are regularly spaced with a frequency predicted by the linear model. As the nonlinearity of pre-rolling friction tends to dominate the system response, the locations of the local minimums and maximums will tend to spread out, leading to the long tails often characteristic of ultra precision settling, (see figure 4). As a final note, precision motion control industry best practices would include feedforward of as many variables as possible. However, since it is desired to compare only the feedback control to an ideal representation of this feedback control, feedforward compensation should not be used in this step.

As an illustrative example, consider the data presented in figures 3 and 4 . The linear approximation predicts a local minimum or maximum about every $8 \mathrm{~ms}$. This approximation holds until the system moves between the velocity reversals at -637 $\mathrm{nm}$ and $115 \mathrm{~nm}$, where the system takes $23 \mathrm{~ms}$ to travel between these points. Pre-rolling effects are even more pronounced between the next set of velocity reversals where it takes $67 \mathrm{~ms}$ to travel between $115 \mathrm{~nm}$ and $-19 \mathrm{~nm}$. Thus, this step response has suggested that the servo behaves rather linearly in settling when velocity reversals are further than $3.8 \mu \mathrm{m}$ from the target location and that there is a pre-rolling feature which is approximately $600 \mathrm{~nm}$ from velocity reversal that significantly affects settling performance. As shown in figure 6, this distance corresponds to the approximate travel required to reach the saturation point in the hysteresis curve, starting from a zero force condition. Note that there is a small offset in the figure that is thought to be due to amplifier bias.

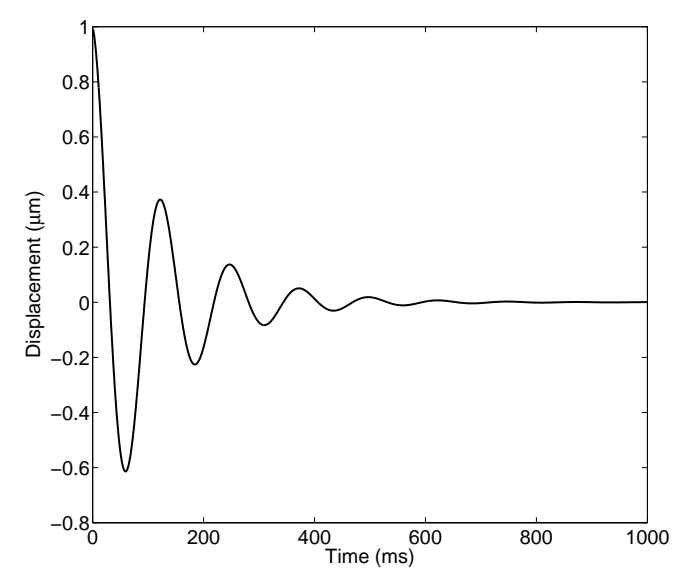

Figure 7: The identification profile consists of a sine wave multiplied by an exponential decay. For this example the sinusoidal component has a frequency of $8 \mathrm{~Hz}$.

To summarize use of the step response as a preliminary identification tool: the locations of local minimums and maximums of a step response are predicted by linear control theory, when these locations deviate from their predictions there is likely a pre-rolling feature with a length scale on the order of the last correctly predicted location. Given the simplicity of this method, a method of automating it will be given in the next section. The deviation of time between predicted time between peaks and actual time between peaks, which constitutes entering into a regime of significant pre-rolling effects, is a tunable parameter. Next, an alternate method of friction parameter identification will be presented.

\subsection{Identification Profile}

Since previous efforts have shown pre-rolling phenomenon to be relatively rate independent [1,32], pre-rolling behavior can also be identified by commanding a relatively slow, motion profile consisting of a decaying sinusoid. This proposed identification profile is thought to be well suited for this application because this profile shape mimics the decaying oscillations that are typical of system settling. However, by using this profile, the information contained in an identification signal can be controlled to a greater extent as compared to simply letting the servo execute settling by itself. Such a profile is shown in figure 7. It is not possible to directly measure the force of friction in an industrial stage without additional instrumentation. However, it is possible to measure the electrical current in the motor. The motor force constant provides a way to approximate the force input to the system, yielding a conversion from amperes to newtons. Knowing the motion of the servo and the input force allows the separation of other force components, such as inertial or viscous components, and allows isolation of the force of friction [28]. Figure 6 shows the force versus displacement results for such an identification profile. As suggested by the step response test, there is definitely a significant pre-rolling friction feature on the order predicted by the step response. To assure that pre-rolling friction is isolated from any other force compo- 
nents, the inertial response of the system can be computed by taking the second derivative of the position signal. Ordinarily, it would be ill advised to use a second derivative of a digital signal but, given the small position resolution of $61 \mathrm{pm}$ and low noise content, this practice works well.

\subsection{Friction Model Parameterization}

In adherence to good engineering practice, the most simple solution that accomplishes a task is usually best. For this case, the Dahl model was found to yield very good fits to the observed data. Thus, more complex models were not explored.

Fitting a friction model to this data is a relatively easy task which can be accomplished by numerous gray-box identification methods. In the case of this effort, the data are fit using a genetic algorithm. Recalling the form of the Dahl model as stated in equation (3), the free parameters are the initial contact stiffness, $\sigma$, the level of Coulomb friction, $F_{C}$, and the shape factor, $i$. As is often common practice, the shape factor is set to $i=1$. This leaves two tunable parameters, $\sigma$ and $F_{C}$.

\section{Identification Study}

In this section, the experimental hardware is introduced. This is followed by the results obtained from executing the identification profile. These results are then compared with an alternate method of identifying the friction model directly from the actual point-to-point motion.

\subsection{Equipment}

The primary piece of equipment used in this study is an Aerotech ALS-130H. The Aerotech ALS130 linear motion stage is one of a family of designs that use crossed-roller bearings along with non-contact linear motors and encoders to achieve nanometer-level positioning resolution over centimeters of travel. The particular stage used in this experiment includes a scale with a $0.004 \mathrm{~mm}$ fundamental period, resulting in a sensor resolution of approximately $61 \mathrm{pm}$ following 16bit $(65536 x)$ signal interpolation. True positioning resolution is on order of nanometers, but difficult to characterize with an acceptable test uncertainty ratio and is the subject of further investigation [33]. The stage provides $100 \mathrm{~mm}$ of linear travel and has a moving mass of $1.8 \mathrm{~kg}$. The stage is driven by an NDrive ML Linear Controller/Drive by Aerotech. This gives a servo sampling rate of $8 \mathrm{kHz}$. Figure 8 shows a picture of the ALS-130H linear stage.

\subsection{Identification Profile}

Following the procedure discussed in the previous section, the identification profile was executed 300 times at base frequencies of $8 \mathrm{~Hz}, 16 \mathrm{~Hz}$, and $32 \mathrm{~Hz}$ along the range of travel of the servo, resulting in 900 data sets. The identification profile started at an amplitude of $1 \mu \mathrm{m}$ in all cases. The identification profile is conducted at various base frequencies to determine if rate dependent effects are present. Table 1 shows the basic statistics of the resulting model fits to the data. Fortunately, this

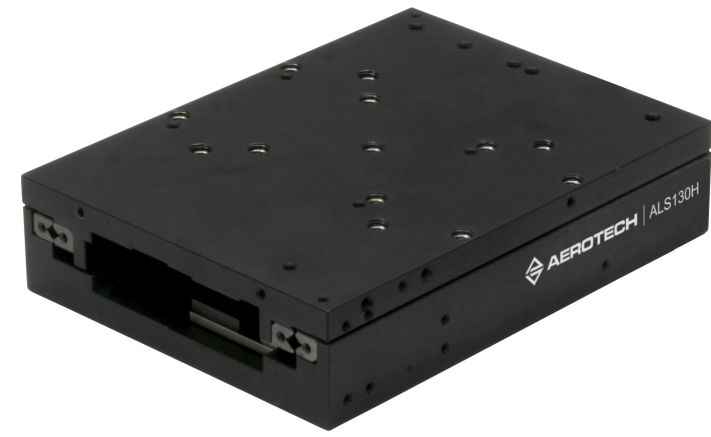

Figure 8: The ALS-130H linear stage, by Aerotech Inc., is the precision servo used in this experimental study.

Table 1: Statistical results of using the identification profile of Figure 7 to identify Dahl friction model parameters.

\begin{tabular}{ccccccccc}
\hline & \multicolumn{2}{c}{$\mathbf{8 H z}$} & & \multicolumn{2}{c}{$\mathbf{1 6 H z}$} & & \multicolumn{2}{c}{$\mathbf{3 2 H z}$} \\
\cline { 2 - 3 } Symbol & $\sigma$ & $F_{C}$ & & $\sigma$ & $F_{C}$ & & $\sigma$ & $F_{C}$ \\
Units & $\mathrm{N} / \mu \mathrm{m}$ & $\mathrm{N}$ & & $\mathrm{N} / \mu \mathrm{m}$ & $\mathrm{N}$ & & $\mathrm{N} / \mu \mathrm{m}$ & $\mathrm{N}$ \\
\hline Mean & 8.23 & 0.61 & & 8.99 & 0.66 & & 8.74 & 0.63 \\
St. Dev. & 0.94 & 0.17 & & 1.70 & 0.23 & & 2.46 & 0.13 \\
Min. & 6.60 & 0.42 & & 3.46 & 0.39 & & 4.47 & 0.39 \\
Max. & 10.39 & 1.14 & & 12.87 & 2.13 & & 15.47 & 0.92 \\
\hline
\end{tabular}

friction data suggests no rate dependent effects but, it does display some apparent variation in the frictional parameters. An attempt was made to correlate this variation to location along the range of travel but, no repeatable results could be produced. This uncertainty was formally considered in the other references [27, 28].

\subsection{Extension to Point-to-Point Motion}

The goal of this section is to determine whether or not model parameterizations similar to those found in the previous step will describe the behavior of the force of friction during servo settling after a step motion. This effort theorizes that, as the servo settles after a step motion, there should be a region where the servo behavior is accurately described by a friction model parameterization similar to those computed in the previous step. It is not known if the system will smoothly transition into the, now familiar, pre-rolling behavior or if some other factors will tend to momentarily dominate the system response.

To investigate this proposition, 250 instances of $5 \mathrm{~mm}$ steps are conducted at the locations where the identification profile studies were performed. The data to be analyzed begins immediately after the motion command concludes and the settling begins. As with the identification profile studies, it is assumed that the only significant dynamics of the system are inertial and frictional. Thus, the inertial component can be subtracted from the system response leaving only the frictional component. Figure 9 shows an example of the resulting frictional response being separated from the inertial response. A similar amplifier offset that was observed in figure 6 is again observed in this 


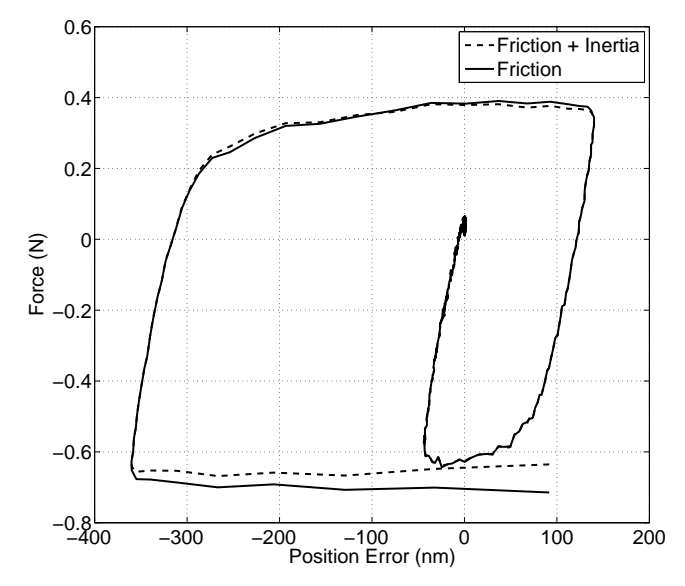

Figure 9: Total and frictional component settling response to a $5 \mathrm{~mm}$ step.

Table 2: Comparison of mean and standard deviation of the Dahl friction parameters from the identification profile tests and the actual step and settle tests.

\begin{tabular}{cccccc}
\hline & \multicolumn{2}{c}{ Identification Profile } & & \multicolumn{2}{c}{ Step Tests } \\
\cline { 2 - 3 } \cline { 5 - 6 } Symbol & $\sigma$ & $F_{C}$ & & $\sigma$ & $F_{C}$ \\
Units & $\mathrm{N} / \mu \mathrm{m}$ & $\mathrm{N}$ & & $\mathrm{N} / \mu \mathrm{m}$ & $\mathrm{N}$ \\
\hline Mean & 8.66 & 0.64 & & 8.73 & 0.58 \\
St. Dev. & 1.84 & 0.18 & & 2.10 & 0.16 \\
Min. & 3.46 & 0.39 & & 6.09 & 0.39 \\
Max. & 15.47 & 2.13 & & 13.76 & 1.16 \\
\hline
\end{tabular}

figure. From figure 9 it is clear that the inertial response of the system during settling, for this servo and this motion profile, tends to become insignificant, particularly as the system executes velocity reversals near the target location. It also appears that the frictional response could be approximated by the Dahl model.

To further investigate this observation the identification algorithm is run on 250 settling data sets. Table 2 shows basic statistics computed over all of the identified parameters from the profile tests and the identified parameters from the actual step and settle tests. When examining the statistics presented in table 2, it is seen that both methods yield very similar identification results. Thus, considering that force of friction versus displacement settling data appears to have the characteristics of the pre-rolling friction model, the settling data and the identification profile data result in statistically similar identified parameters. Further, the fits of the pre-rolling friction models to the settling data are as good, if not better, than the identification profile data. Thus, it appears that the system does enter the pre-rolling regime upon completing a rapid step motion.

Although both friction identification methods produced similar results in the work presented, it is expected that the identification profile method would provide the best performance in general. In the case of using a different servo mechanism or a different motion profile, the frictional behavior may not be as clearly isolated at the beginning of the settling process. The identification profile method also provides some flexibility in constructing the identification data in a form that would be most useful. Finally, the use of the identification profile could provide a better method to identify potential rate dependence.

\section{Point-to-Point Motion Experiments}

The rapid point-to-point motion experiments are divided into two distinct sets of experiments. The first set of experiments used a factory tuned PID controller without acceleration feedforward as a baseline for comparison. The second set of experiments used a high performance PID tuning constructed by an industry expert control system engineer with $10+$ years of experience. In addition to this aggressive tuning, tuned acceleration feedforward was also implemented. The second case was designed to represent the practical limits of conventional linear control system best practices. In all tests settling to tolerances of $\pm 3 \mathrm{~nm}$ to $100 \mathrm{~nm}$ will be studied. The $\pm 3 \mathrm{~nm}$ minimum settling tolerance was selected because it is the listed amount of expected peak-to-peak in-position noise for a comparable servo.

\subsection{First Experiment}

The first PID tuning should not be thought of as bad or flawed but, only as less aggressive. Depending on the design of the servo, task of the mechanism, and payload, such a tuning may actually be a high performance tuning. Figure 2 shows the loop transmission plot for this particular tuning. The move studied in this experiment is a $5 \mathrm{~mm}$ step with acceleration and deceleration rate of $1 \mathrm{~m} / \mathrm{s}^{2}$. In the center of the step a maximum velocity of approximately $70 \mathrm{~mm} / \mathrm{s}$ is achieved and the motion profile takes $143 \mathrm{~ms}$ to complete. This test consists of 250 steps conducted on a $25 \mathrm{~mm}$ section of the device travel, (25 passes, 5 steps up, 5 steps back). In this parameterization of the NIASA compensator $\tau_{D}=0.01 \mathrm{~s}, \sigma=4 \mathrm{~N} / \mu \mathrm{m}$, and $F_{C}=0.6 \mathrm{~N}$. Note that half the average contact stiffness, $\sigma$, was used since values that are less than or lower than average are associated with increased stability [27].

Figure 10 shows the mean time to settle to tolerances of \pm 3 $\mathrm{nm}$ to $100 \mathrm{~nm}$ for the first PID tuning, the same tuning combined with the NIASA compensator. Dashed lines have been added to figure 10 to indicate one standard deviation in settling time as computed across the data set. For this range of settling tolerances, this controller tuning, and this particular motion profile, servo settling time is reduced by NIASA control by between $80.5 \%$ and $87.4 \%$ for all settling tolerances.

Examining a few time series plots of the position error signal also proves interesting. Figure 11 shows three randomly selected time series of position error during settling for the PID controller. The common feature amongst each of these series is that the system appears to be significantly slowed down at each velocity reversal that occurs. This behavior is identical to that seen in figure 4 and exemplifies how pre-rolling friction reduces servo settling performance. With this performance baseline in consideration the NIASA settling compensator is now considered.

In examining time series of the position error figure 12 shows the algorithm settling the system in a manner that is very close 


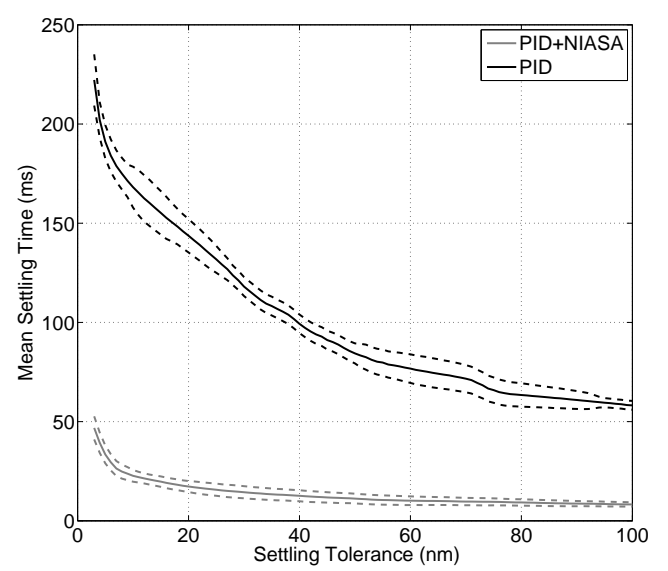

Figure 10: Comparison of mean settling time for steps $5 \mathrm{~mm}$ with standard PID controller and PID with NIASA compensator (dashed lines indicate one standard deviation).

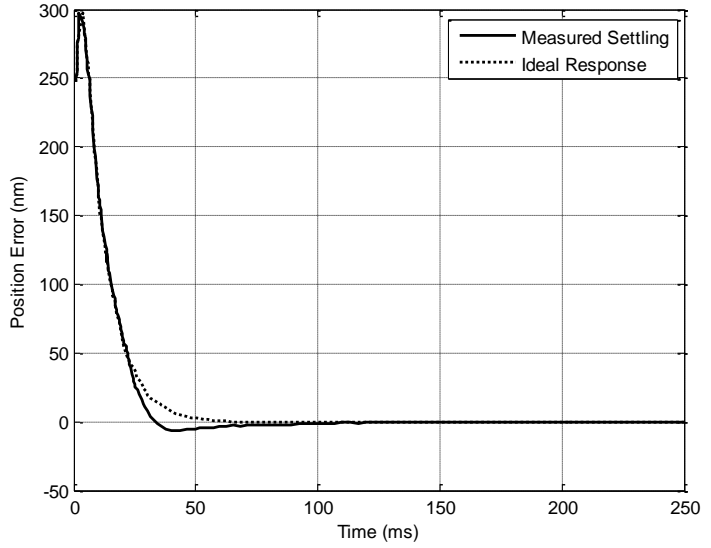

Figure 12: Comparison of settling response of PID+NIASA control with ideal first order response of $\tau=0.01 \mathrm{~s}$.

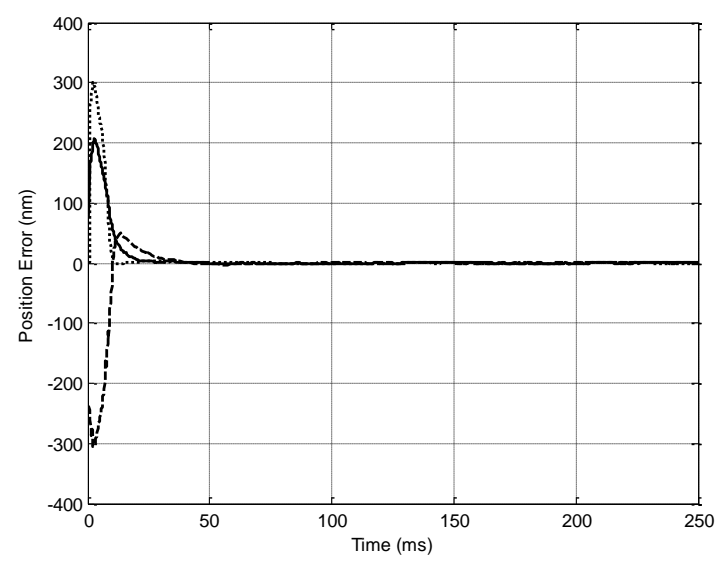

Figure 13: Three random examples of system response for PID+NIASA control are less ideal, but still show rapid convergence.

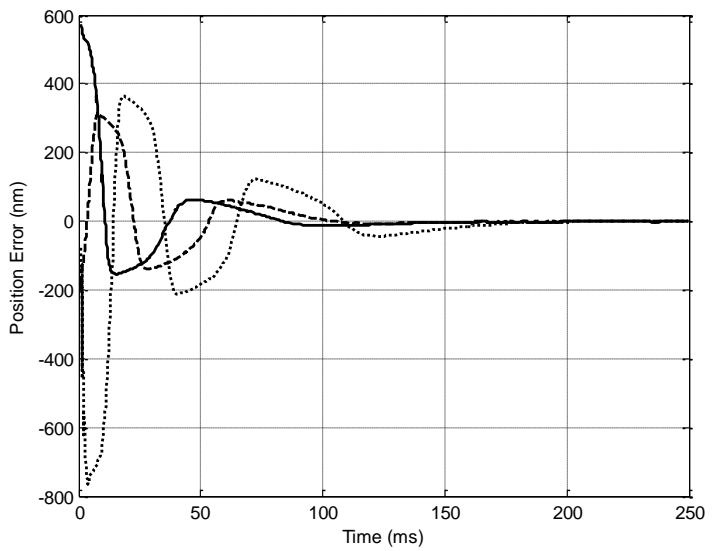

Figure 11: System settling response for three random trials under PID control alone, clearly showing pre-rolling friction effects. to how the proposed theory suggested that it should be done $[27,28]$. Apart from a slight undershoot, this settling profile looks very much like a first order decay. Not all settling responses proved to be as clean and ideal as those shown in figure 12 but, the algorithm proved to be surprisingly resilient and their time responses also prove to be interesting. Figure 13 shows the settling responses for the same sequence of randomly selected moves presented in figure 11. As this data suggests, it is not uncommon for overshoots and undershoots to occur during the settling process even with the NIASA compensator. However, compared to the original PID tuning, the NIASA compensator appears to be effective at quickly turning the system around when velocity reversals occur in the final few hundred nanometers of settling.

This experiment shows that the NIASA compensator works quite well to reduce system settling time when used with this particular controller tuning. However, one can still make the valid argument that, by using higher control gains, the effects of pre-rolling friction can be minimized. While it is true to stiffer control will reduce pre-rolling effects, there is a practical limit 

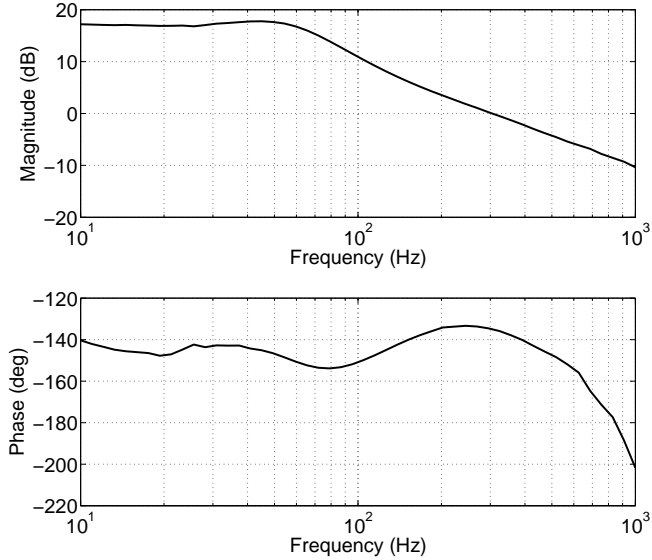

Figure 14: Loop transmission plot for the second PID controller showing the system to have a cross-over frequency near $315 \mathrm{~Hz}$ and a phase margin of about 50 degrees.

to the amount of stiffness that can be added to the system, and it is still possible that pre-rolling effects will be significant even with the stiffest feasible controller. A second valid argument is, properly tuned acceleration feedforward control of the moving mass should leave the system much closer to the target location when the motion profile ends. There is always some measure of uncertainty within the system and some small changes are continuously occurring within the system. Thus, consistent and reliable nanometer precision is not likely to be possible using only feedforward methods. Taking a realistic perspective of the point-to-point motion task, it would be very nice for the system to be within a few hundred nanometers or less of the final target location but, this region is also exactly where pre-rolling effects tend to dominate the system response. Thus, final servo settling, done by conventional PID methods could still suffer from the same problems. This discussion raises a very interesting question: Could a high bandwidth control combined with properly tuned feedforward, now coupled with the NIASA compensator, be used to achieve even better point-to-point performance?

\subsection{Second Experiment}

In contrast to the first PID tuning, this high performance tuning should not be thought of as the best tuning for all devices in all situations. It just happens that this was the best tuning that could be done on a very simple, yet well designed servo, with no payload. Figure 14 shows the loop transmission plot for this particular tuning. The move studied in this experiment is also a $5 \mathrm{~mm}$ step but, with acceleration and deceleration rate of $10 \mathrm{~m} / \mathrm{s}^{2}$ (the maximum rate of acceleration of this device). In the center of the step a maximum velocity of approximately $223.5 \mathrm{~mm} / \mathrm{s}$ is achieved and the motion profile takes $46 \mathrm{~ms}$ to complete. This test consists of 500 steps conducted on a $25 \mathrm{~mm}$ section of the device travel, (50 passes, 5 steps up, 5 steps back). In this parameterization of the NIASA compensator $\tau_{D}=0.01 \mathrm{~s}$, $\sigma=4 \mathrm{~N} / \mu \mathrm{m}$, and $F_{C}=0.6 \mathrm{~N}$.

Figure 15 shows the mean time to settle to tolerances of \pm 3 $\mathrm{nm}$ to $\pm 100 \mathrm{~nm}$ for the high performance PID controller with

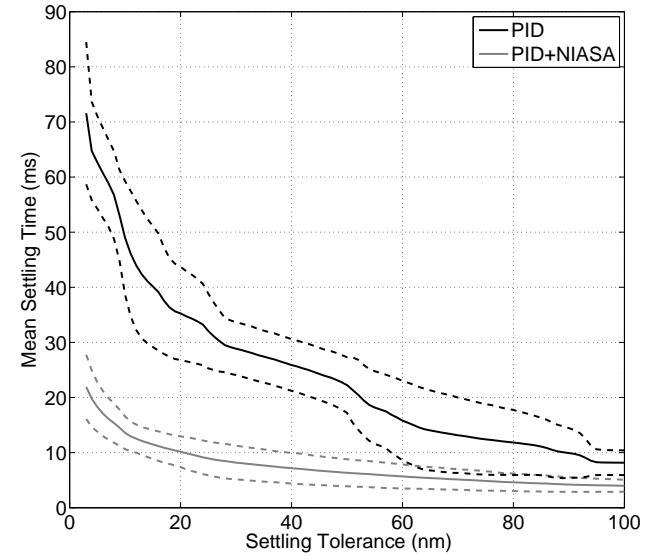

Figure 15: Comparison of high performance PID + feedforward compensation with and without NIASA compensator for $5 \mathrm{~mm}$ steps (dashed lines are one standard deviation).

Table 3: Analysis of the root mean square in-position error shows that all of the controller configurations have very similar in-position characteristics.

\begin{tabular}{ccc}
\hline Controller & & In-Position Noise $\left.\mathbf{( n m}_{r m s}\right)$ \\
Regular PID & & 0.48 \\
Regular PID + NIASA & 0.50 \\
High Perf. PID & & 0.49 \\
High Perf. PID + NIASA & 0.48 \\
\hline
\end{tabular}

feedforward and also this same tuning with the addition of the NIASA compensator. Dashed lines have been added to figure 15 to indicate one standard deviation in settling time as computed across the data set. For this range of settling tolerances, this controller tuning, and this particular motion profile, servo settling time is reduced by between $50.5 \%$ and $73.0 \%$ for all settling tolerances. Thus, it appears that the NIASA settling compensator has the ability to make improvements to even a very high performance PID controller with properly tuned feedforward compensation.

\subsection{In-Position Noise}

There was some concern that the adjustment of system gains done with the NIASA compensator could increase the inposition noise of the system. To quantify the in-position characteristics, the root mean square of the position error $500 \mathrm{~ms}$ after settling to $\pm 3 \mathrm{~nm}$ was analyzed for all of the steps in each test. The results shown in table 3 show that there is no significant change in the in-position characteristics of the servo with or without the settling compensation being active.

\subsection{Small Step Experiments}

At the beginning of this effort, the targeted application for this control algorithm was a step motion of several millimeters with a settling tolerance of a few nanometers. Recalling the previous discussion of how some compensation methods are only useful in specific situations, to answer the question of when the NIASA compensator is useful, additional tests are 


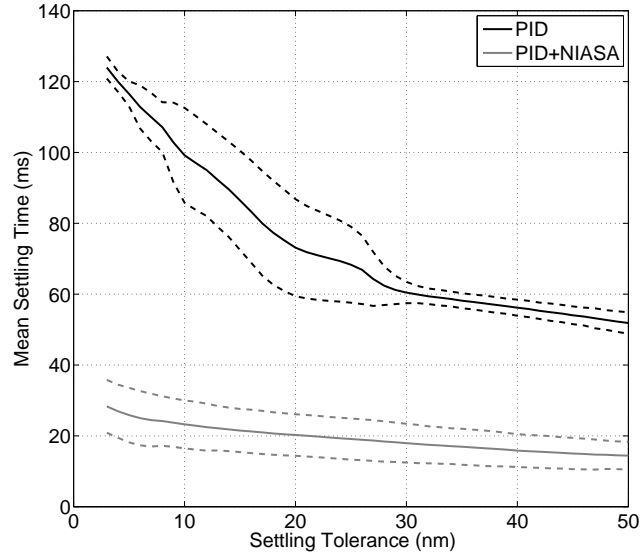

Figure 16: Comparison of mean settling time for $5 \mu \mathrm{m}$ steps with standard PID controller and PID with NIASA compensator (dashed lines indicate one standard deviation).

conducted. In the first set of tests, where the $5 \mathrm{~mm}$ move is used, the servo moves well outside the pre-rolling regime for the gross motion of the step. As this occurs, it is safe to assume that the system experiences fully developed rolling friction before the settling phase. Thus, testing larger displacement step motions is not interesting because they would involve nearly the same frictional conditions as the $5 \mathrm{~mm}$ step. However, testing of smaller displacement steps, motions that just leave the pre-rolling regime or possibly remain entirely in the pre-rolling regime, would constitute a frictional scenario that has not yet been experimentally explored.

The small step experiments consist of 500 instances of $5 \mu \mathrm{m}$ steps and 500 instances of $50 \mathrm{~nm}$ steps. The tests will be performed with the standard PID controller and the high performance PID controller for cases when the NIASA compensator is active and when the NIASA compensator is disabled. These step tests will use a similar format as the previous test, five steps in one direction followed by five steps in the returning direction. The $5 \mu \mathrm{m}$ steps use an acceleration and deceleration rate of $100 \mathrm{~mm} / \mathrm{s}^{2}$, which leads to a maximum commanded velocity of approximately $698 \mu \mathrm{m} / \mathrm{s}$ and a motion profile lasting $15 \mathrm{~ms}$. The $50 \mathrm{~nm}$ steps use an acceleration and deceleration rate of 1 $\mathrm{mm} / \mathrm{s}^{2}$. This yields a maximum commanded velocity of 6.98 $\mu \mathrm{m} / \mathrm{s}$ and a motion profile lasting $15 \mathrm{~ms}$.

The $5 \mu \mathrm{m}$ step represents a case where the gross motion of the step may take the system just outside the pre-rolling regime and into nearly fully developed rolling friction. In this experiment the mean time to settle to $\pm 3 \mathrm{~nm}$ to $\pm 50 \mathrm{~nm}$ is calculated. Figures 16 and 17 show the settling performance of the system for the standard PID controller and the high performance controller respectively. As seen in figures 16 and 17, the NIASA compensator is effective in reducing servo settling times for step motions that are just outside the pre-rolling regime, when combined with either PID controller.

The $50 \mathrm{~nm}$ step test is designed to show a case where the step motion may not exit the pre-rolling regime. Once again, the use of the NIASA compensator is able to significantly re-

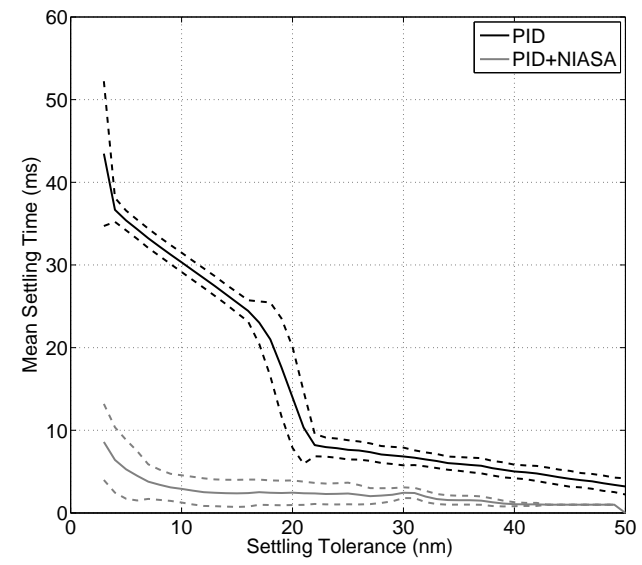

Figure 17: Comparison of high performance PID + feedforward compensation with and without NIASA compensator for $5 \mu \mathrm{m}$ steps (dashed lines are one standard deviation)

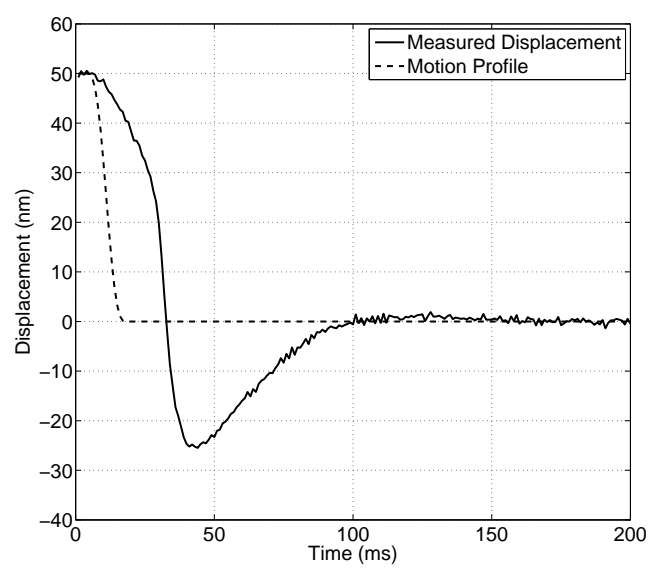

Figure 18: Typical $50 \mathrm{~nm}$ step with standard PID control.

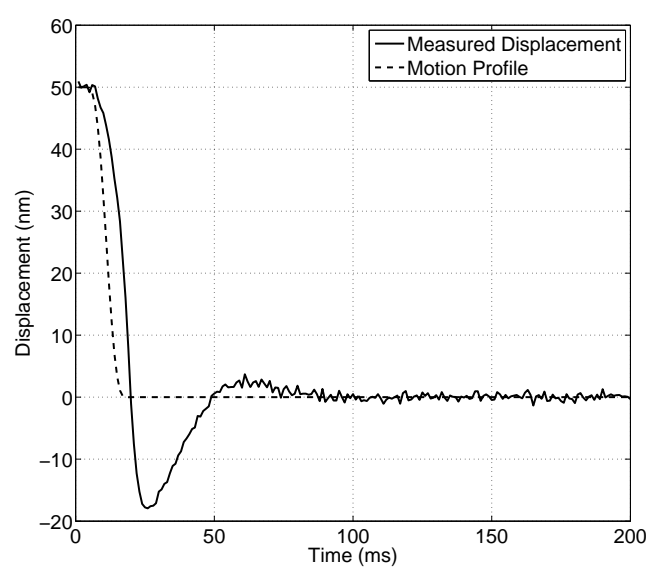

Figure 19: Typical $50 \mathrm{~nm}$ step with high performance PID control. 


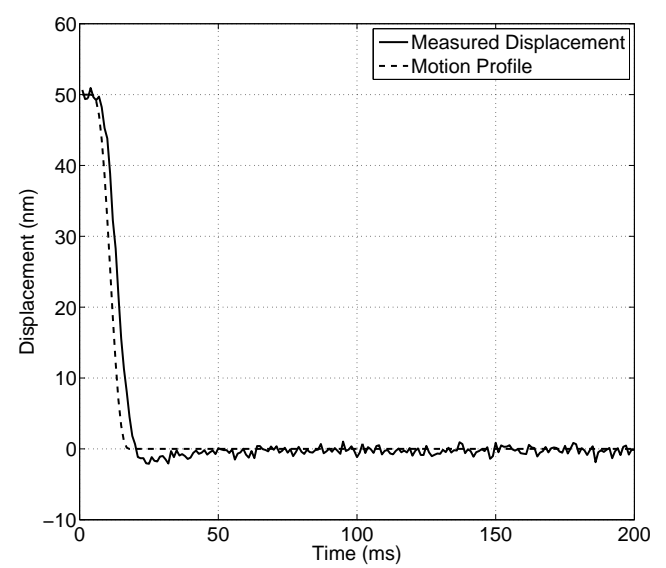

Figure 20: Typical $50 \mathrm{~nm}$ step with standard PID control and NIASA compensator.

duce servo settling time. The most interesting data from this experiment is the temporal response of the system. Figures 18 and 19 show examples of typical servo motion for the standard PID controller and the high performance PID controller. In both cases, there appears to be a delay in the system response to the motion profile that is followed by an overshoot in the intended position. When the NIASA compensator is added to the system, as shown in figure 20, this delay in the system response is reduced and the overshoot is eliminated. Figure 20 shows typical responses when the NIASA compensator is combined with the standard PID controller. A plot for the NIASA compensator combined with the high performance PID controller is not shown because it is visually indistinguishable from figure 20. As compared to either PID controller, the NIASA compensator appears to significantly reduce the position error when tracking the gross motion of the step. This interesting result suggests that future efforts should be made in exploring the utility of this methods for servo tracking applications and that future efforts could evaluate the NIASA compensator as a global method of controlling systems subject to friction.

\section{Frequency Domain Analysis}

Frequency domain analysis, such as loop transmissions, are well understood by control system engineers. Extensions of this concept to nonlinear systems are easy to construct, offering a quasi-linear interpretation of system behavior. While these tools are not the final word on performance and stability when a system is not linear, they do provide some insight into system behavior and it would be useful to have some understanding of the outputs of such tools when applied to precision servos. Further, applying the loop transmission tool to the NIASA compensator helps to show how the compensator deceases servo settling time.

In this study, swept-sine system identification is conducted on the servo with the NIASA compensator enabled and with it disabled. All of the tests are conducted sequentially and
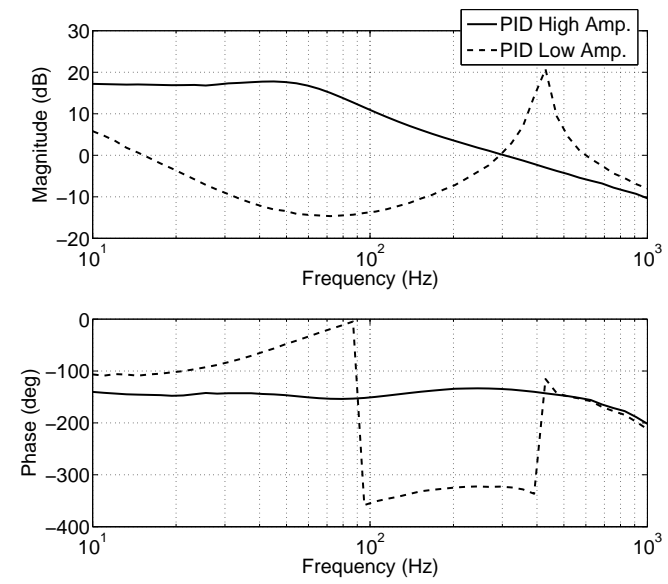

Figure 21: Comparison of high amplitude and low amplitude loop transmissions for PID controller.

in the same location on the device travel to minimize uncontrolled factors. The PID controller used for comparison and also in conjunction with the NIASA compensator is what will be known as the high performance tuning. This tuning is used because it best showcases the limitations of linear control best practices. Two amplitudes of excitation are given to the system in separate tests, a large amplitude excitation, where the system leaves the pre-rolling regime, and a small amplitude excitation, where the system remains in the pre-rolling regime. For an in depth theoretical explanation of many of the features seen in the loop transmissions, it is suggested to read Helmick and Messner [34].

Figure 21 shows typical loop transmissions for high amplitude and low amplitude inputs. For the high amplitude input, which would probably be used in conventional tuning practices, the system has a frequency cross over at $315 \mathrm{~Hz}$. However, when the low amplitude excitation is applied, the frequency cross over is reduced to $15 \mathrm{~Hz}$ and a resonant peak appears near $400 \mathrm{~Hz}$. This is because, at low amplitude excitation, pre-rolling friction appears to have the effect similar to adding a stiff spring to the system. Although these two test cases do not provide a global description of the frictional process in of themselves, it is intuitive that a system will not settle as quickly as might be expected if the frequency cross over suddenly changed from 315 $\mathrm{Hz}$ to $15 \mathrm{~Hz}$.

In the NIASA compensator loop transmission tests, the design time constant, $\tau_{d}$, is set to $0.01 \mathrm{~s}$ for all trials. The system has shown a mean value of $\sigma=8.66 \mathrm{~N} / \mu \mathrm{m}$ and $\sigma=8.73 \mathrm{~N} / \mu \mathrm{m}$ for the identification profile method and the step test method, respectively, and a minimum identified value for $\sigma=3.46$ $\mathrm{N} / \mu \mathrm{m}$. The simulation results done in previous work suggest that system stability is assured when $\sigma$ is taken to be less than the identified value $[27,28]$. Thus, in the first trial the conservative value of $\hat{\sigma}=4 \mathrm{~N} / \mu \mathrm{m}$ is used. To study the effect of changing this parameter, a second trial is conducted with the more aggressive value of $\hat{\sigma}=8 \mathrm{~N} / \mu \mathrm{m}$.

Figure 22 shows the system response to high and low amplitude excitation with the NIASA compensator enabled and 

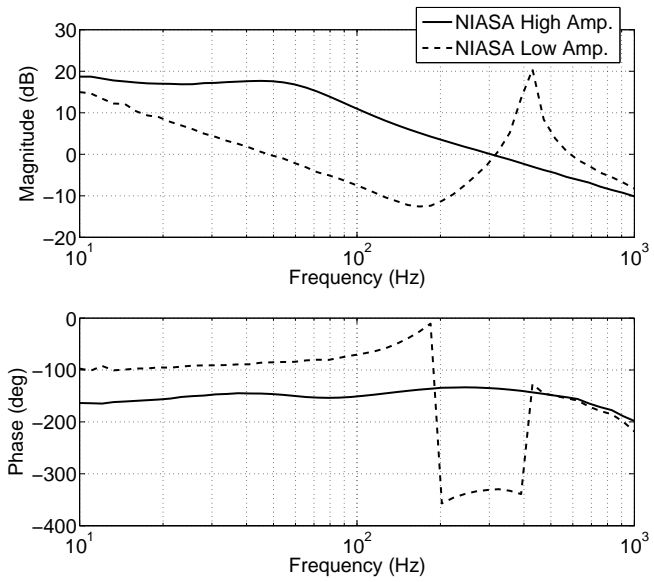

Figure 22: Comparison of high amplitude and low amplitude loop transmissions for PID+NIASA with $\hat{\sigma}=4$.
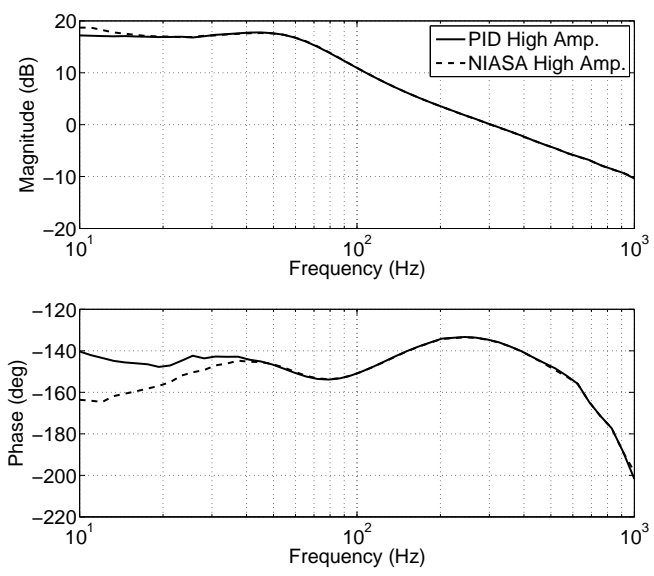

Figure 23: Comparison of high amplitude loop transmissions for PID and PID+NIASA with $\hat{\sigma}=4$.

$\hat{\sigma}=4 \mathrm{~N} / \mu \mathrm{m}$. One key characteristic is that the cross over frequency of the low amplitude case has been increased to about $50 \mathrm{~Hz}$ as compared to the $15 \mathrm{~Hz}$ seen in figure 21. However, when doing a side by side comparison of the high amplitude inputs to the system with only PID control and PID with the NIASA compensator, as shown in figure 23 , it is found that there is very little difference in the loop transmission response. This is desirable since the original, PID, high amplitude loop transmission is a good system response.

In a second NIASA compensator experiment, the friction model is changed so that $\hat{\sigma}=8 \mathrm{~N} / \mu \mathrm{m}$. The low amplitude loop transmission for this case is compared to that of the $\hat{\sigma}=4$ $\mathrm{N} / \mu \mathrm{m}$ case in figure 24 . It is interesting to see that doubling the contact stiffness parameter used in the model adds about $6 \mathrm{~dB}$ to the response at low frequencies (since stiffness was doubled) and the frequency crossover is increased to about $90 \mathrm{~Hz}$. With this increase of low amplitude bandwidth the high amplitude loop transmission still remains very similar to the original PID controller, as shown in figure 25.

Given the nature of these frequency domain tests, it was not
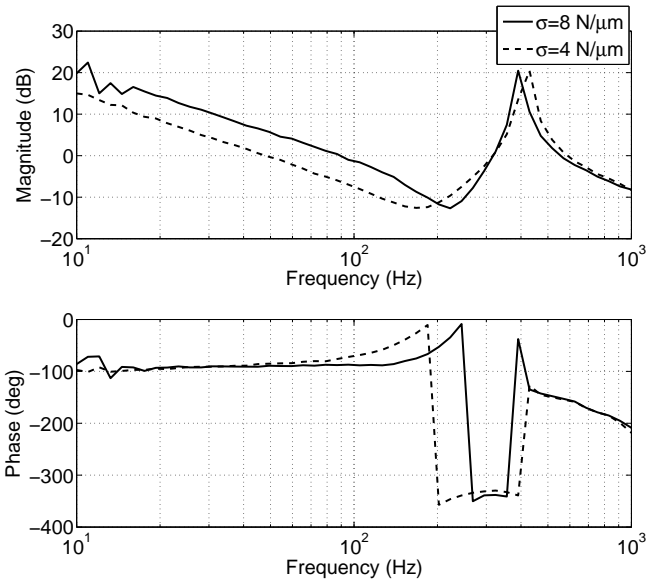

Figure 24: Comparison of low amplitude loop transmissions for PID+NIASA with $\hat{\sigma}=4$ and $\hat{\sigma}=8$.
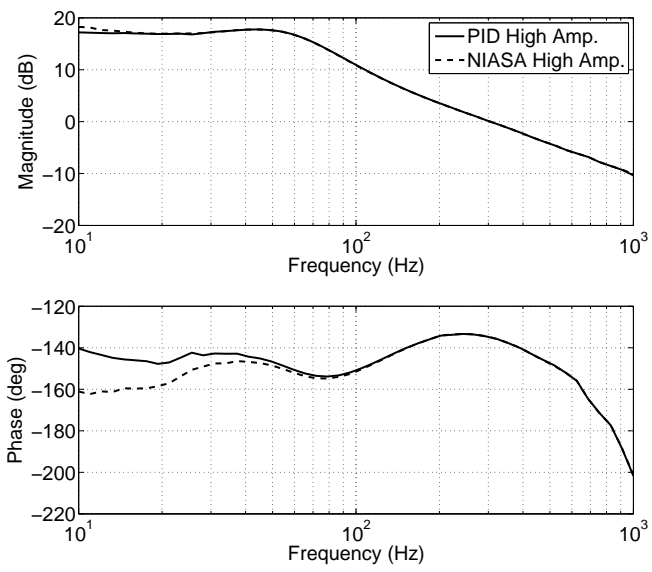

Figure 25: Comparison of high amplitude loop transmissions for PID+NIASA with $\hat{\sigma}=8$. 
possible to analyze displacement versus force data, as was done in the previous friction identification studies. Thus, it was not possible to determine the frictional parameters of the system in these studies. It is interesting to see that the NIASA compensator with $\hat{\sigma}=4 \mathrm{~N} / \mu \mathrm{m}$ leads to a frequency cross over near $50 \mathrm{~Hz}$ and $\hat{\sigma}=8 \mathrm{~N} / \mu \mathrm{m}$ lead to a frequency cross over near 90 $\mathrm{Hz}$. Recall that the concept behind the NIASA compensator is to design a first-order-like system response, at least for low frequencies, where inertia is small. Assuming that the initial contact stiffness was near the observed mean value of either 8.66 $\mathrm{N} / \mu \mathrm{m}$ or $8.73 \mathrm{~N} / \mu \mathrm{m}$ and that the design time constant was $0.01 \mathrm{~s}$, using $\hat{\sigma}=4 \mathrm{~N} / \mu \mathrm{m}$ led to an effective time constant of $0.02 \mathrm{~s}$, about half as fast as the design time constant, while using a $\hat{\sigma}$ value that was probably half of the true $\sigma$ value. Using $\hat{\sigma}=8$ $\mathrm{N} / \mu \mathrm{m}$ lead to an effective time constant of $0.011 \mathrm{~s}$, slightly less than the design time constant, and the value of $\hat{\sigma}$ was probably slightly less than the true value of $\sigma$. Thus, the frequency domain observations tend to also agree with the proposed theory.

In summary, the NIASA compensator, when used in conjunction with PID control, has been shown to increase the bandwidth of control for small amplitude motion while preserving the desirable behavior already seen in loop transmission studies at large amplitude motion. The frequency domain observations provided useful insight into how the NIASA compensator is able to reduce servo settling time.

\section{Conclusion}

The proposed NIASA compensator worked very well to increase ultra precision servo settling performance. In some cases, this method was able to reduce servo mean settling time by up to $87.4 \%$. This effort succeeded in constructing a linear model of the servo mechanism and identifying where this approximation tends to break down because of frictional nonlinearities. Through a series of small displacement tests and identification procedures, pre-rolling friction is characterized for the servo mechanism. The identified characteristics of pre-rolling friction are found to generalize to observations from real pointto-point motion data. Thus, the potential problems, which occur during system settling, are captured in the identified pre-rolling friction models.

A feedback control algorithm, called the Nonlinear Integral Action Settling Algorithm (NIASA), is designed based on the identified friction model. The NIASA compensator is developed to be robust to parametric uncertainty in the pre-rolling friction process, as compared to other friction compensation methods that rely heavily on model accuracy or are only analyzed in idealized cases. The NIASA compensator is designed to be used in conjunction with conventional control system best practices of properly tuned feedforward control and PID feedback control.

Some basic frequency domain analysis of the behavior of the NIASA compensator is presented to provide some familiar perspective how the NIASA compensator functions. This analysis shows that the NIASA compensator amounts to a method of increasing low frequency gains for small motions, where prerolling friction dominates system response. For large motion, where the linear components of the system dominate, the desirable frequency domain characteristics are preserved.

In a case study with a reasonably tuned PID controller, servo settling times to \pm 3 to $\pm 100 \mathrm{~nm}$, are reduced by between $80.5 \%$ and $87.4 \%$. As a secondary case study a high performance PID controller was tuned by an experienced control system engineer to represent the practical limits of linear control methods. When the NIASA compensator is used, servo settling time is still reduced by between $50.5 \%$ and $73.0 \%$. Although the NIASA compensator was designed to increase settling performance for relatively large point-to-point motions, similar positive results are achieved when the method is applied to smaller step motions. Further, the algorithm appears enhance the ability of the servo mechanism to track nanometer scale point-to-point motion profiles. Thus, experimental data suggests that the NIASA compensator is a viable solution for dealing with friction in ultra precision point-to-point motion for a wide variety of step sizes. The technique should be applicable to other applications with friction that follow a Dahl type model (e.g. sliding friction). Additionally, the NIASA compensator may prove to be a feasible method of increasing tracking performance of nanometer scale motion profiles.

\section{Acknowledgments}

This work has been supported by Aerotech Inc. The authors would also like to thank Aerotech Inc. for providing the experimental hardware and baseline PID tunings.

\section{Disclosure Statement}

This work has been supported by Aerotech Inc. The first three authors received research support from Aerotech. The last author is an employee of Aerotech, Inc.

\section{Role of the Funding Source}

The research was nearly solely conducted by the University of Pittsburgh authors at the University of Pittsburgh. The author from Aerotech (Steve Ludwick) had a minor contribution by implementing the high-performance PID tuning with acceleration feedforward). It was mutually agreed that we should publish the findings, given that they were very favorable.

\section{References}

[1] P. Dahl, A solid friction model, Tech. rep., Space and Missile Systems Organization Air Force Systems Command (1968).

[2] C. C. de Wit, H. Olsson, K. Åstrom, P. Lischisnsky, A new model for control of systems with friction, IEEE Transactions on Automatic Control 40 (3) (1995) 419-426.

[3] J. Swevers, F. Al-Bender, C. Ganseman, T. Prajogo, An integrated friction model structure with improved presliding behavior for accurate friction compensation, IEEE Transactions on Automatic Control 45 (2000) 675689.

[4] B. Bucci, D. Cole, J. Vipperman, S. Ludwick, Friction modeling of linear rolling element bearings in high precision linear stages, in: Proceedings of the American Society for Precision Engineering, 2009. 
[5] H. Olsson, K. Åstrom, C. C. de Wit, M. Gafvert, P. Lischinsky, Friction models and friction compensation, European Journal of Control 4 (1998) $176-195$.

[6] F. Altpeter, M. Grunenberg, P. Myszkorowski, R. Longchamp, Autotuning of feedforward friction compensation based on the gradient method, Proceedings of the American Control Conference.

[7] S. Chen, K. Tan, S. Huang, Improvement of tracking performance of servomechanical system by an accurate four-parameter friction modelling and compensation, IEEE Control Systems Magazine (2007) 28-34.

[8] Z. Jamaludin, H. Van Brussel, J. Swevers, Friction compensation of a xy feed table using friction model-based feedforward and an inverse-modelbased disturbance observer, IEEE Transactions on Industrial Electronics PP (99) (2009) 1-1.

[9] V. Lampaert, J. Swevers, F. Al-Bender, Experimental comparison of different friction models for accurate low-velocity tracking, in: Proceedings of the 10th Mediterranean Conference on Control and Automation, 2002.

[10] D. Rizos, S. Fassois, Friction identification based upon the LuGre and Maxwell slip models, IEEE Transactions on Control Systems Technology 17 (2009) 153-161.

[11] F. Altpeter, Friction modeling, identification, and compensation, Ph.D. thesis, Ecole Polytechnique Federale de Lausanne (1999).

[12] T. Tjahjowidodo, F. Al-Bender, H. V. Brussel, W. Symens, Friction characterization and compensation in electro-mechanical systems, Journal of Sound and Vibrations 308 (2007) 632-646.

[13] C. C. de Wit, P. Lischinsky, Adaptive friction compensation with partially known dynamic friction model, International Journal of Adaptive Control and Signal Processing 11 (1997) 65-80.

[14] H. Olsson, K. Åstrom, Observer-based friction compensation, in: Proceedings of the 35th Conference on Decision and Control, 1996.

[15] H. Olsson, Control of systems with friction, Ph.D. thesis, Lund Institute of Technology (1996).

[16] F. Altpeter, P. Myszkorowski, M. Kocher, R. Longchamp, Friction compensation: PID synthesis and state control, in: European Control Conference, 1997.

[17] U. Schafer, G. Brandenburg, Model reference position control of an elastic two-mass system with backlash and Coulomb friction using different types of observers, Power Electronics and Motion Control 3 (1990) 797801.

[18] G. Brandenburg, U. Schafer, Influence and partial compensation of simultaneously acting backlash and Coulomb friction in a speed and position controlled, elastic two-mass system, in: Proceedings of the International Conference on Electrical Drives, 1988.

[19] A. Dixit, S. Suryznarayanan, Adaptive observers for servo systems with friction, 17th IEEE International Conference on Control Applications.

[20] J. Martinez-Rosas, L. Alvarez-Icaza, Adaptive compensation of dynamic friction in an industrial robot, in: 17th IEEE International Conference on Control Applications, 2008.

[21] I. Nilkhamhang, A. Sano, Adaptive friction compensation using the GMS model with polynomial Stribeck function, Proceedings of International Conference on Control Applications.

[22] G. Brandenburg, U. Schafer, Influence and adaptive compensation of simultaneously acting backlash and Coulomb friction in elastic two-mass systems of robots and machines tools, in: International Conference of Control and Applications, 1989.

[23] C. Walrath, Adaptive bearing friction compensation based on recent knowledge of dynamic friction, Automatica 20 (1984) 717-727.

[24] S. Futami, A. Furutani, S. Yoshida, Nanometer positioning and its microdynamics, Nanotechnology 1 (1990) 31-37.

[25] S. Yoshida, K. Iizuka, Approaches to nanometer technology: a review of the Yoshida nano-mechanism project, Tech. rep., Research and Development Corporation of Japan (1990).

[26] B. Bucci, D. Cole, J. Vipperman, S. Ludwick, Practical friction compensation for ultra precision point-to-point motion, in: Plenary Presentation and Proceedings of the American Society for Precision Engineering, 2010 .

[27] B. Bucci, A practical method for friction compensation in rapid pointto-point motion, Ph.D. thesis, University of Pittsburgh, Department of Mechanical Engineering and Material Science (2011).

[28] B. Bucci, D. Cole, V. J. S. Ludwick, S., A nonlinear control algorithm for reducing settling time in high-precision point to point motion, IEEE Transactions on Control System Technology.
[29] T. Tjahjowidodo, Characterization, modelling, and control of mechanical systems comprising material and geometric nonlinearities, Ph.D. thesis, Department Werktuigkunde Katholieke Universiteit Leuven (2006).

[30] J. Adams, S. Payandeh, Methods for low-velocity friction compensation: Theory and experimental study, Journal of Robotic Systems 13 (1996) 391-404.

[31] D. Helmick, W. Messner, Simultaneous control of a precision linear stage in multiple lubrication modes with the complex lag, in: 2008 American Control Conference, 2008.

[32] F. Al-Bender, V. Lampaert, J. Swevers, The generalized Maxwell-slip model: A novel model for friction simulation and compensation, IEEE Transactions on Automatic Control 50 (2005) 1883-1888.

[33] R. E. Fesperman, M. A. Donmez, S. P. Moylan, Ultra-precision linear motion metrology of a commercially-available linear translation stage, in: Proceedings of the 26th Annual Meeting of the American Society for Precision Engineering, 2011.

[34] D. Helmick, W. Messner, Describing function analysis of Dahl model friction, in: 2009 American Control Conference, 2009. 


\section{List of Figures}

1 Example of the type of response seen from Dahl's friction model to a decaying sinusoidal motion. . . . . . . . .

2 A loop transmission plot for the first PID controller shows the system to have a cross-over frequency near $100 \mathrm{~Hz}$ and a phase margin of about 35 degrees. . . . . . . . . . . . . . . . . . . . . . . 3

3 Comparison of actual $100 \mu \mathrm{m}$ step response to that predicted by a linear model.

4 Final micrometer of settling demonstrating that the system response is dominated by the nonlinearity of pre-rolling

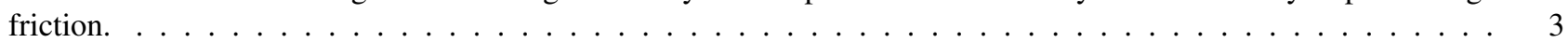

5 Loop transmission curves for various drive ratios of the plant. . . . . . . . . . . . . . . 4

6 Force versus displacement curve generated by the identification profile. . . . . . . . . . . . . . 5

7 The identification profile consists of a sine wave multiplied by an exponential decay. For this example the sinusoidal component has a frequency of $8 \mathrm{~Hz} \ldots \ldots \ldots \ldots$

8 The ALS-130H linear stage, by Aerotech Inc., is the precision servo used in this experimental study. . . . . . . . . 6

9 Total and frictional component settling response to a $5 \mathrm{~mm}$ step. . . . . . . . . . . . . . . . . . . . 7

10 Comparison of mean settling time for steps $5 \mathrm{~mm}$ with standard PID controller and PID with NIASA compensator

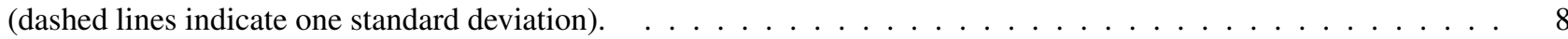

11 System settling response for three random trials under PID control alone, clearly showing pre-rolling friction effects.

12 Comparison of settling response of PID+NIASA control with ideal first order response of $\tau=0.01 \mathrm{~s}$. . . . . . 8

13 Three random examples of system response for PID+NIASA control are less ideal, but still show rapid convergence. 8

14 Loop transmission plot for the second PID controller showing the system to have a cross-over frequency near 315 $\mathrm{Hz}$ and a phase margin of about 50 degrees. . . . . . . . . . . . . . . . . . . . . . .

15 Comparison of high performance PID + feedforward compensation with and without NIASA compensator for 5 mm steps (dashed lines are one standard deviation) $\ldots \ldots \ldots \ldots \ldots$

16 Comparison of mean settling time for $5 \mu \mathrm{m}$ steps with standard PID controller and PID with NIASA compensator (dashed lines indicate one standard deviation) . . . . . . . . . . . . . . . . . . . .

17 Comparison of high performance PID + feedforward compensation with and without NIASA compensator for 5 $\mu \mathrm{m}$ steps (dashed lines are one standard deviation) $\ldots \ldots \ldots \ldots$

18 Typical $50 \mathrm{~nm}$ step with standard PID control. . . . . . . . . . . . . . . . . . . . . . . 10

19 Typical $50 \mathrm{~nm}$ step with high performance PID control. . . . . . . . . . . . . . . . . . . . . . 10

20 Typical $50 \mathrm{~nm}$ step with standard PID control and NIASA compensator. . . . . . . . . . . . . . . . . 11

21 Comparison of high amplitude and low amplitude loop transmissions for PID controller. . . . . . . . . . . . . 11

22 Comparison of high amplitude and low amplitude loop transmissions for PID+NIASA with $\hat{\sigma}=4$. . . . . . . 12

23 Comparison of high amplitude loop transmissions for PID and PID+NIASA with $\hat{\sigma}=4 \ldots \ldots$. . . . . . . . . . 12

24 Comparison of low amplitude loop transmissions for PID+NIASA with $\hat{\sigma}=4$ and $\hat{\sigma}=8$. . . . . . . . . . . . 12

25 Comparison of high amplitude loop transmissions for PID+NIASA with $\hat{\sigma}=8 \ldots \ldots \ldots$

\section{List of Figures}




\section{List of Tables}

1 Statistical results of using the identification profile of Figure 7 to identify Dahl friction model parameters. . . . . . 6

2 Comparison of mean and standard deviation of the Dahl friction parameters from the identification profile tests and the actual step and settle tests. . . . . . . . . . . . . . . . . . . . . . . . .

3 Analysis of the root mean square in-position error shows that all of the controller configurations have very similar

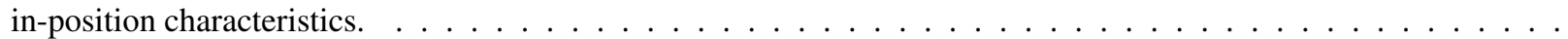

